\title{
Transcriptomic study of lipopolysaccharide-induced sepsis damage in a mouse heart model
}

\author{
CUNRONG CHEN $^{1 *}$, JUNTING WENG $^{2 *}$, DEXIANG FANG ${ }^{2 *}$, JIANFEI CHEN $^{2}$ and MIN CHEN ${ }^{2}$ \\ ${ }^{1}$ Department of Critical Care Medicine, Union Hospital Affiliated to Fujian Medical University, Fuzhou, Fujian 350000; \\ ${ }^{2}$ Department of Critical Care Medicine, Affiliated Hospital of Putian University, Putian, Fujian 351100, P.R. China
}

Received January 29, 2019; Accepted April 29, 2020

DOI: $10.3892 /$ etm.2020.9086

\begin{abstract}
Sepsis is an emergency systemic illness caused by pathogen infection and the combined result of the underactivity and overactivity of a patient's own immune system. However, the molecular mechanism of this illness remains largely unknown. Lipopolysaccharide (LPS) was injected to establish a sepsis model, and heart tissue was used to analyze transcriptome changes in mice. LPS injection was used to develop a sepsis model, which resulted in cardiac tissue rearrangement and inflammatory response activation. An RNA-sequencing-based transcriptome assay using mouse heart tissue with or without LPS injection showed that 3,326 and 1,769 genes were upregulated and downregulated, respectively ( $>2$-fold changes; $\mathrm{P}<0.05$ ). Furthermore, these differentially expressed genes were classified into 20 pathways, including 'Wnt signaling pathway', 'VEGF signaling pathway' and 'TGF- $\beta$ signaling pathway', and these altered genes were enriched in 41 Gene Ontology terms. The application of Wnt3a inhibited the activation of the LPS-induced inflammatory response and activated Wnt signaling, as well as protecting against LPS-mediated cardiac tissue damage in mice. In contrast, inhibition of Wnt signaling by injection of its inhibitor IWR induced plasminogen activator inhibitor-1 expression and resulted in cardiac structure derangement, which was similar to the symptoms caused by injection of LPS, suggesting that LPS-induced damage to heart tissue may be via inhibition of Wnt signaling. The present analyses showed that Wnt signaling serves a pivotal role in sepsis development and may improve our understanding of the molecular basis underlying sepsis.
\end{abstract}

Correspondence to: Dr Min Chen, Department of Critical Care Medicine, Affiliated Hospital of Putian University, 999 Dongzhen East Road, Putian, Fujian 351100, P.R. China

E-mail: chenminicu@ptu.edu.cn

*Contributed equally

Key words: transcriptome, lipopolysaccharide, Wnt, sepsis, heart

\section{Introduction}

Sepsis is an emergency systemic illness that is due to the combined effects of the under- and overactivity of the immune systems of patients. Initial symptoms of sepsis include multiple organ impairment and can possibly result in severe secondary infections. The immune systems of patients react to infection as a response to high febricity, decreased blood pressure and organ dysfunction, which was defined in the 1970s-1980s (1). In the mid-last century, a previous study on microbiology and immunology showed that several hallmarks of infectious diseases were not only generally caused by the body's own immune response but also by invading pathogens (2). Previously, life-threatening sepsis was shown to result in organ dysfunction; the definition of the new Sepsis-3 indicates a $60 \%$ possibility of mortality within 28 days (3-6).

Bacterial endotoxins, including lipopolysaccharide (LPS), are potential inducers of inflammation. These endotoxins induce a complex pathogen-host interaction that enables the host to respond to infection and results in hyperinflammation. LPS affects innate and acquired immune responses in several systems, such as the nervous, respiratory, circulatory, endocrine and metabolic systems (7). Inflammation of the vessels and endocardium, and persistent activation of inflammatory signals are the initial signs of cardiac dysfunction in sepsis (8). The role and molecular mechanism of inflammatory signal transduction in the myocardial injury of sepsis are important to elucidate the molecular mechanism of this disease.

Molecular research has further hinted at the underlying mechanisms of sepsis. A genome-wide association (GWA) study and high-throughput omic technologies have facilitated studies on the diagnosis of sepsis by screening sepsis-specific molecular indicators as biomarkers. Common sepsis biomarkers include high-mobility group box 1, C-reactive protein, tumor necrosis factor (TNF)- $\alpha$, interleukin (IL)-1, IL-6, IL-10 and macrophage migration inhibitory factor (9-16). GWA revealed that the FER tyrosine kinase (FER) gene serves an important role in intercellular signaling in patients with sepsis (17), as well as vacuolar protein sorting 13 homolog A and cysteine rich secretory protein LCCL domain containing 2, which may be involved in survival after 28 days (18). Methylthioadenosine is a sepsis biomarker that is involved in the inflammatory response and is related to high rates of fever-induced host cell death (19). Several previous studies have shown that 
sepsis is associated with the inflammatory response in the heart $(8,20,21)$, but the detailed molecular mechanism remains unclear. The Wnt and inflammatory response pathways were associated with $\mathrm{T}$ cell-specific transcription factor (TCF)/lymphoid enhancer-binding factor (LEF) $(22,23)$. The typical Wnt signaling pathway, also known as the $\beta$-catenin pathway or $\beta$-catenin/TCF pathway (24), affects a wide array of signaling channels (25-27). A major characteristic of the $\mathrm{WNT} / \beta$-catenin pathway includes the stabilization of cytosolic $\beta$-catenin. Before stimulation, $\beta$-catenin is phosphorylated by a destruction complex that contains glycogen synthase kinase-3 $\beta$ (GSK3 $\beta)$; phosphorylated $\beta$-catenin becomes ubiquitinated and is degraded by the proteasome $(23,28)$. The stimulation of Wnt signaling reduces GSK $3 \beta$ activity, thereby resulting in the accumulation of $\beta$-catenin in the nucleus, which results in the transcription of Wnt target genes by TCF/LEF to regulate biological processes, such as cell proliferation, differentiation and survival (23). Furthermore, Wnt signaling has been reported to be associated with inflammatory response signaling to control disease (28), but whether Wnt signaling is involved in sepsis development has not yet been investigated.

In the present study transcriptome changes were analyzed using heart tissues from LPS-injected mice as a sepsis model. Furthermore, the differentially expressed genes were classified by Gene Ontology (GO) and Kyoto Encyclopedia of Genes and Genomes (KEGG) terms. Among the pathways enriched, Wnt signaling was further examined for its roles in the regulation of the inflammatory responsive pathway and cardiac tissue. The present results might provide a theoretical basis for improving the clinical treatment protocol of sepsis in the future.

\section{Materials and methods}

Ethics statement. Animals were obtained from The Laboratory Animal Center of Fuzhou Wushi Animal Center. All animal studies were performed according to the National Institutes of Health Guide Concerning the Care and Use of Laboratory Animals (29) with the approval of the Animal Experimentation Ethics Committee of The Affiliated Hospital of Putian University.

Animal model treatments. A total of 90 female C57BL/6 mice (age, 8 weeks; weight, 220-250 g) were purchased from The Nanjing Animal Center. All mice were housed in cages in an SPF animal room with a temperature of $20 \pm 2^{\circ} \mathrm{C}$ and a humidity of $55 \pm 5 \%$ under a 12 -h light/dark cycle. All rats had free access to food and water. The animals received LPS in sterile PBS by intraperitoneal injection. In total, 50 of the 90 mice were divided into the following five groups $(n=10)$ : Control group (intraperitoneal injection with $0.9 \%$ saline solution) and four LPS treatment groups (intraperitoneal injection with LPS at $50 \mathrm{mg} / \mathrm{kg}$ body weight for 1, 2, 3 and 4 days).

The remaining 40 mice were used to investigate whether the Wnt signaling is involved in LPS-induced heart damage. In the treatment group, mice weighing 220-250 g were injected with LPS (50 mg/kg body weight), LPS + Wnt3a (100 ng/kg body weight) or IWR (100 ng/kg body weight). Mice in the control group were administered an equivalent volume of vehicle (DMSO) for $1 \mathrm{~h}$ before LPS injection. Mice with matched ages, sexes and weights were randomly divided into the normal control group (10 mice), the LPS model group (10 mice), LPS+Wnt3a group (10 mice) and IWR-injected group (10 mice). LPS from Escherichia coli serotypes 0111:B4, Wnt3a and IWR were all purchased from Sigma-Aldrich; Merck KGaA. The mice were sacrificed by an intraperitoneal injection of sodium pentobarbital $(150 \mathrm{mg} / \mathrm{kg})$ following the AVMA Guidelines for the Euthanasia of Animals (30). Death was then confirmed by a certain set of criteria: Respiratory arrest, cardiac arrest, dilation of the pupils and disappearance of nerve reflex.

Histopathological observation of damaged myocardial tissue. The heart samples were taken immediately after 4 days of DMSO, LPS, Wnt3a or IWR injection, and were subsequently fixed in $10 \%$ formaldehyde solution for $20 \mathrm{~min}$ at room temperature. The tissue was dehydrated, and paraffin embedded, and pathological sections were cut at $4-\mu \mathrm{M}$ thickness. Hematoxylin and eosin (HE) staining for $20 \mathrm{~min}$ at room temperature was performed to analyze the pathological events of the myocardium under a light microscope at x200 magnification to examine heart muscle arrangement.

RNA isolation and cDNA reverse transcription. Ground heart tissues (30 min post stimulation) were preserved in TRIzol ${ }^{\circledR}$ reagent (Invitrogen; Thermo Fisher Scientific Inc.) at $-80^{\circ} \mathrm{C}$ until use. Total RNA isolation was performed by following the TRIzol ${ }^{\circledR}$ manufacturer's protocol with an RNeasy Mini kit (Qiagen, Inc.) for RNA purification. DNase I. SuperScript III First-Strand Synthesis SuperMix (Thermo Fisher Scientific, Inc.) was used for reverse transcription-quantitative PCR (RT-qPCR) to produce cDNA from $2 \mu \mathrm{g}$ total RNA. The reaction protocol consisted of $25^{\circ} \mathrm{C}$ for $5 \mathrm{~min}, 42^{\circ} \mathrm{C}$ for $60 \mathrm{~min}$ and $70^{\circ} \mathrm{C}$ for $15 \mathrm{~min}$.

$R T-q P C R$. Total RNA was extracted using TRIzol ${ }^{\circledR}$ reagent (Invitrogen; Thermo Fisher Scientific, Inc.) according to the manufacturer's protocol. Subsequently, the selected results from RNA-sequencing (RNA-seq) were verified by RT-qPCR analysis. cDNA was produced using a Prime Script ${ }^{\mathrm{TM}}$ RT-qPCR kit (Takara Bio, Inc.) according to the manufacturer's protocol. qPCR was performed using SYBR ${ }^{\circledR}$ Premix Ex Taq ${ }^{\mathrm{TM}}$ (Takara Bio, Inc.) on a 7900HT fast RT-qPCR instrument (Applied Biosystems; Thermo Fisher Scientific, Inc.) with technical triplicates. The gene-specific primers used for RT-qPCR are listed in Table I. Thermocycling conditions were as follows: Initial denaturation at $95^{\circ} \mathrm{C}$ for $5 \mathrm{~min}$, followed with 35 cycles at $95^{\circ} \mathrm{C}$ for $30 \mathrm{sec}$ and $60^{\circ} \mathrm{C}$ for $30 \mathrm{sec}$. The relative gene expression levels were determined using the $2^{-\Delta \Delta \mathrm{Cq}}$ method (31).

Sequencing analysis. The RNA was extracted using RNAiso Reagent (Takara Biotechnology Co., Ltd.) according to the manufacturer's instructions. The concentration and purity of RNA were determined by Agilent 2100 Bioanaylzer (Agilent Technologies, Inc.). All the samples had RNA integrity numbers of $>8.5$ and concentration $>200 \mathrm{ng} / \mu \mathrm{l}$. Sequencing libraries were generated using TIANSeq Fast RNA Library Kit for Illumina ${ }^{\circledR}$ (cat. no. NR102; Tiangen Biotech Co., Ltd.) following the manufacturer's protocols. After completing the library, paired-end (150 bp) sequencing of the cDNA 
Table I. Primer sequences for reverse transcription PCR.

\begin{tabular}{lll}
\hline Gene & Forward Primer sequences, 5'-3' & Reverse Primer sequences, 5'-3' \\
\hline FZD8 & TCTACAACCGCGTCAAGACC & GGCCGTTCCGGGTACTTAAA \\
$P A I-1$ & AGGCTGGTCCTCGTTAATGC & CACAGAGAGCTGAGCCAACA \\
$I L-8$ & TGTGTGCATAGCCATGTGGT & GAACAGCATGATGAGCAGCG \\
$F G F 21$ & AGCATACCCCATCCCTGACT & TCCTCCCTGATCTCCAGGTG \\
\hline
\end{tabular}

$F Z D 8$, frizzled class receptor 8; PAI-1, plasminogen activator inhibitor 1; $I L-8$, interleukin 9; FGF21, fibroblast growth factor 21.
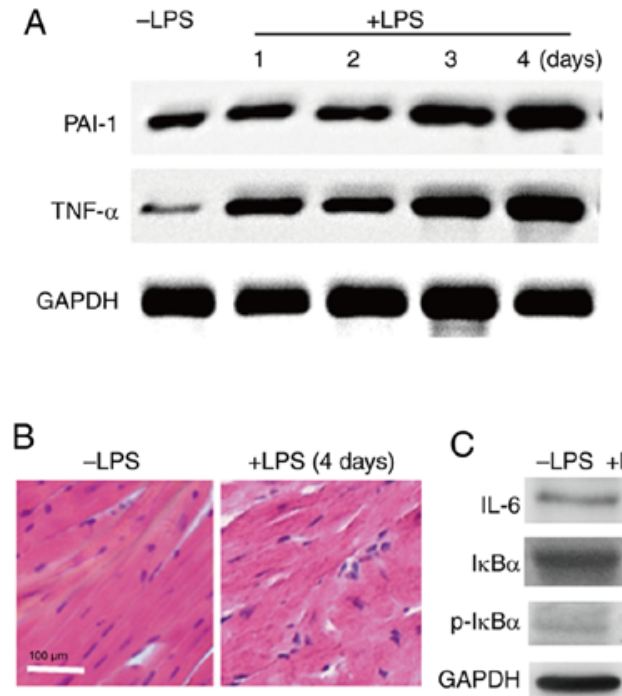
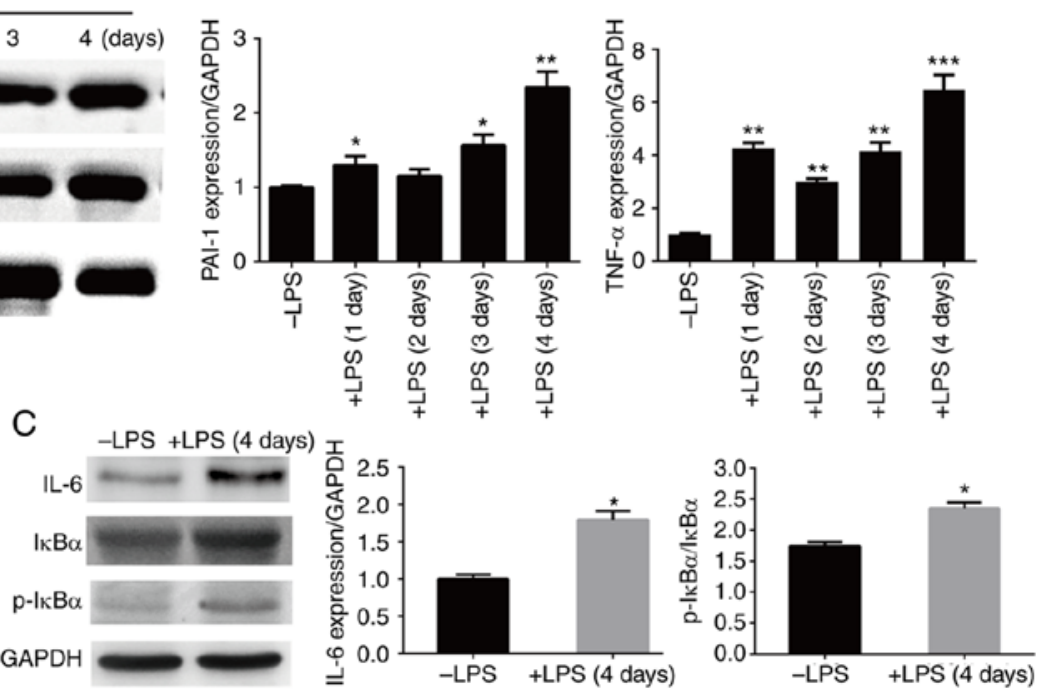

Figure 1. Effects of LPS on heart tissue structure and inflammation response marker levels. (A) Western blot analysis was performed to evaluate the levels of PAI-1 and TNF- $\alpha$ in the heart tissues after 1,2,3 and 4 days of LPS injection. GAPDH was used as a loading control. (B) Histopathology of heart tissues with or without LPS injection for 4 days was assessed by staining tissues with hematoxylin and eosin under a light microscope. scale bar, $100 \mu \mathrm{m}$. (C) Western blot analysis was performed to evaluate the levels of IL-6, IкB $\alpha$ and $\mathrm{p}$-IкB $\alpha$ in the heart tissues with or without LPS injection for 4 days. GAPDH was used as a loading control. ${ }^{*} \mathrm{P}<0.05,{ }^{* * *} \mathrm{P}<0.01,{ }^{* * * *} \mathrm{P}<0.001$ vs. the control group. LPS, lipopolysaccharide; PAI-1, plasminogen activator inhibitor 1 ; TNF- $\alpha$, tumor necrosis factor- $\alpha$; HE, Hematoxylin eosin; IL-6, interleukin 6 ; IкB $\alpha$, inhibitor of nuclear factor $\kappa \mathrm{B}$ kinase $\alpha$; p, phosphorylated.

libraries $(10 \mathrm{nM})$ was performed on the GAIIx instrument (Illumina, Inc.) using the reagents provided in the TruSeq PE Cluster Kit v5-CS-GA (cat. no. PE-203-5001; Illumina, Inc.). A standard analysis protocol was performed (32), and sequencing data were aligned to the hg19 human genome using TopHat version 2.06 (33) and Bowtie2 2.0.0 (34) and mapped to Ensembl transcripts (http://www.ensembl.org). NovelBio Bio-Pharm Technology Co., Ltd. (http://www. novelbio.com/) performed the library construction and sequence using LPS-treated and untreated mouse heart samples.

Analysis of GO categories and KEGG analysis. Differentially expressed genes were classified into diverse biological processes according to the $\mathrm{GO}$ terms (35). GO categories were assessed using a one-tailed Fisher's exact test with a P-value, and corrected for using the false discovery rate (FDR) (36). $\mathrm{P}<0.05$ was considered as significant. $\mathrm{GO}$ enrichment analysis was also performed using a Fisher's exact test using $2 \times 2$ contingency tables (37). As the enrichment increases, the corresponding function becomes more specific. Pathway analysis focuses on the significance of KEGG (http://www. kegg.jp/) (38), Biocarta (http://biocarta.com) (39) and Reatome (http://www.reactome.org) (40). Benjamini-Hochberg multiple testing was used further to correct the Fisher's exact test results, and the threshold of significance was defined as P-value $<0.05$ and FDR $<0.05$ (41).

Western blot analysis. RIPA lysis buffer (Takara Bio, Inc.) supplemented with complete protease inhibitor cocktail (Roche Diagnostics) was used to lyse the cells. The mouse heart tissues were ground in liquid nitrogen, and protein was extracted by adding cold RIPA lysis buffer. A Bradford reagent protein assay kit (Bio-Rad Laboratories, Inc.) was used to determine protein concentrations using bovine serum albumin as the control. Subsequently, a total of $30 \mu \mathrm{g}$ sample protein was loaded per lane and separated using SDS-PAGE on a $10-20 \%$ gel, then subsequently transferred PVDF membranes. The membranes were blocked with buffer $(5 \%$ skimmed milk in $20 \mathrm{mM}$ Tris- $\mathrm{HCl}, 150 \mathrm{mM}$ $\mathrm{NaCl}, 0.1 \%$ Tween-20) for $1 \mathrm{~h}$ at room temperature and incubated with primary antibodies at $4^{\circ} \mathrm{C}$ overnight; The 

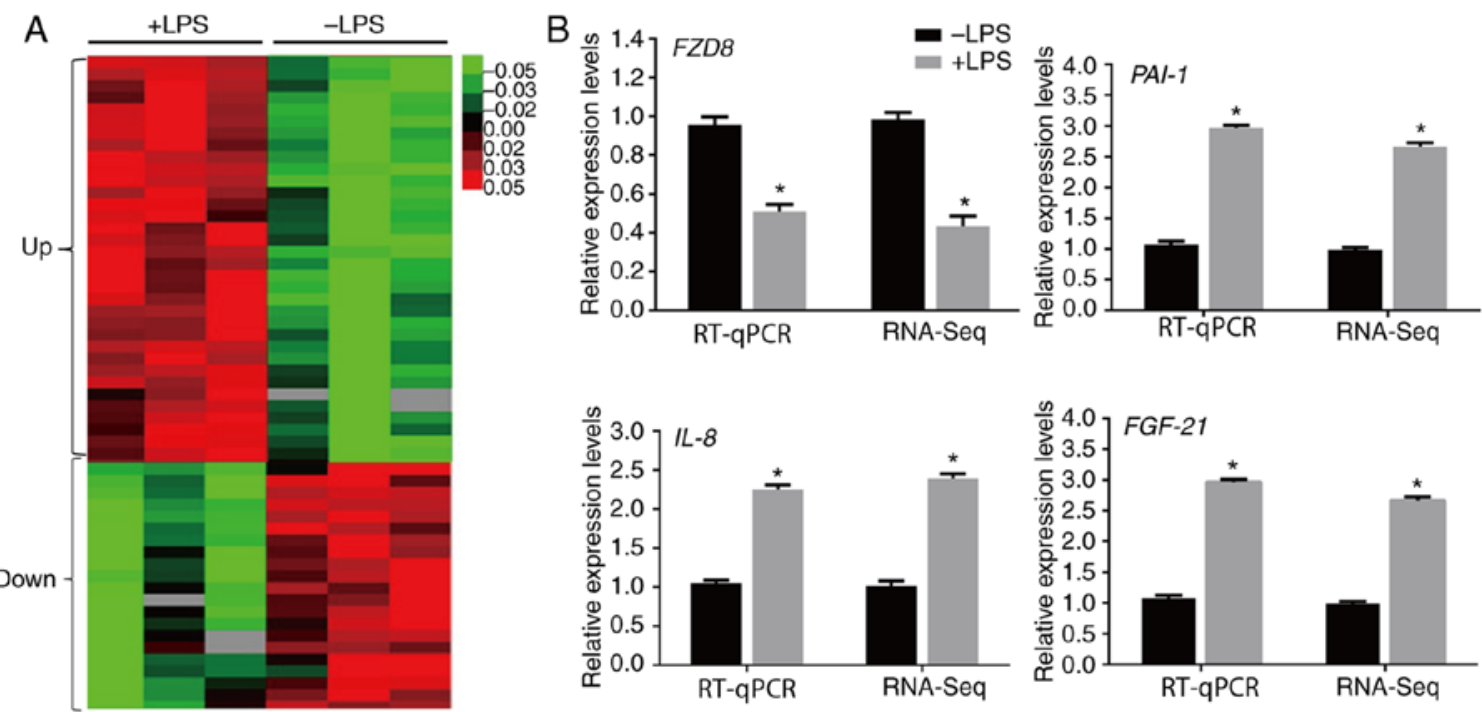

Figure 2. LPS-regulated transcriptome profile in heart. (A) Heat map representing the genes with expression levels that were altered after 4 days of LPS treatment in the mouse heart. Gene expression is shown with a pseudocolor scale, where red denotes higher gene expression levels and green denotes lower gene expression levels $(\mathrm{P}<0.05)$. (B) RT-qPCR was performed to verify the expression levels of $F Z D 8, I L-8, P A I-1$ and $F G F-21$, and the data were compared with the RNA-sequencing data. GAPDH was used as the internal control. "P<0.05 vs. the control group. LPS, lipopolysaccharide; RT-qPCR, reverse transcription-quantitative PCR; FZD8, frizzled class receptor 8; IL-8, interleukin 8; PAI-1, plasminogen activator inhibitor 1; FGF21, fibroblast growth factor 21.

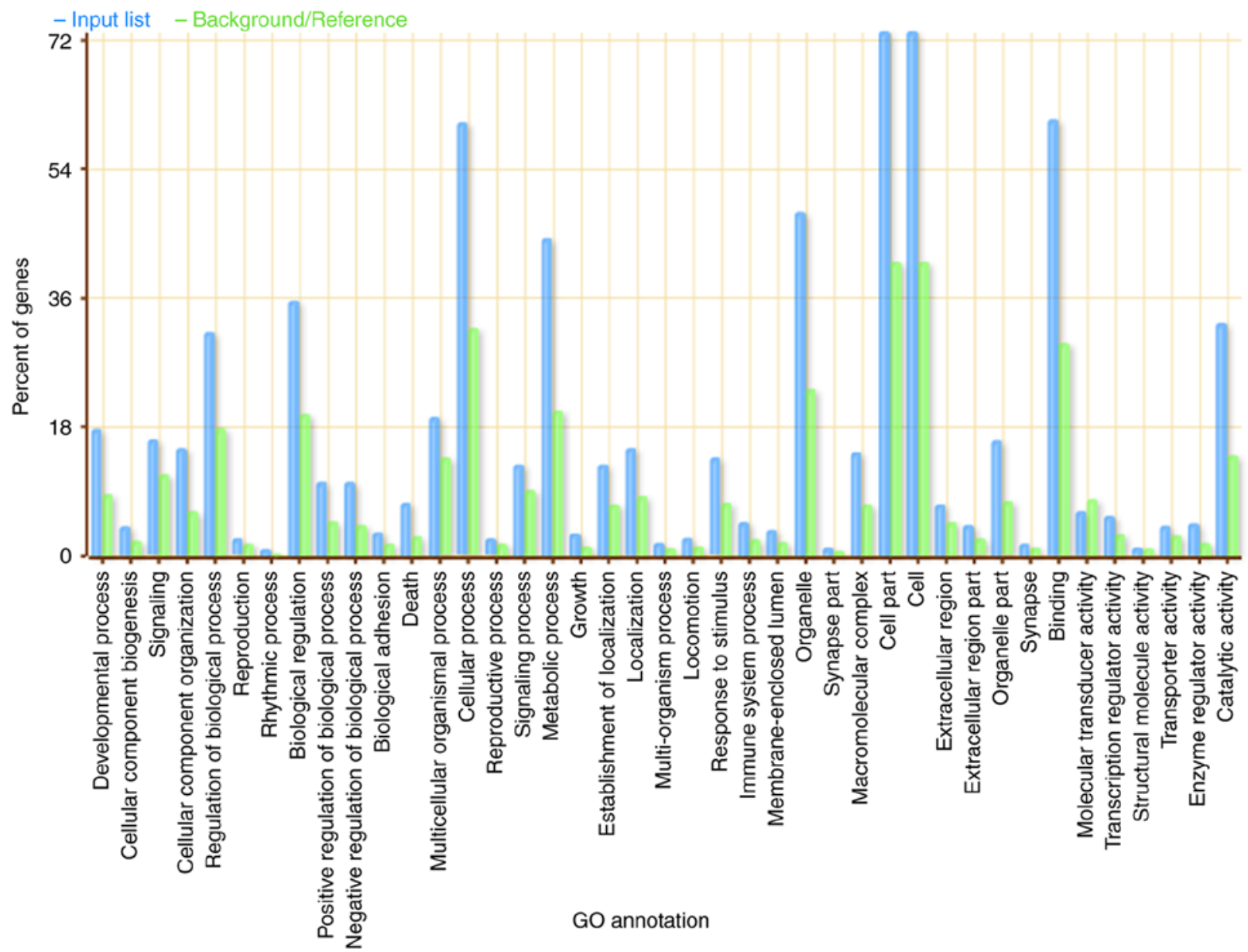

Figure 3. Classification of differentially expressed genes using GO terms. A total of 41 GO terms, including 'developmental process', 'cell part' and 'binding' biological processes, were enriched. GO, Gene Ontology.

primary antibodies used were anti-IL-6 antibody (1:1,000 dilution; cat. no. ab7737; Abcam), anti-TNF- $\alpha$ antibody
(1:1,000 dilution; cat. no. ab11564; Abcam), anti-PAI-1 (1:1,000 dilution; cat. no. ab222754; Abcam), anti-IкB $\alpha$ 


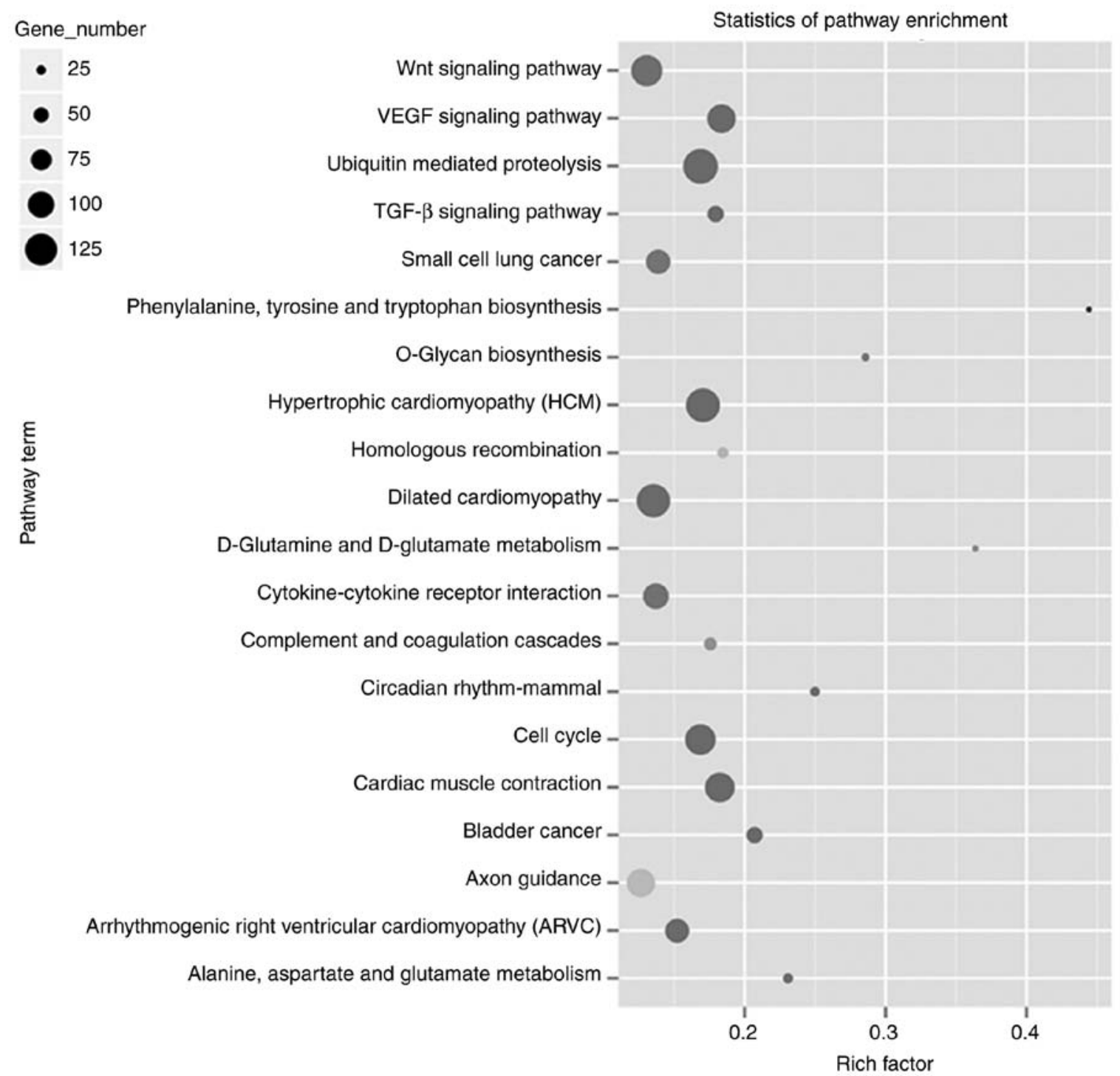

Figure 4. Pathway analysis of differentially expressed genes. The differentially expressed genes under the stimuli of LPS were primarily classified into 20 signaling pathways, which include the Wnt signaling pathway, cell cycle and TGF- $\beta$ signaling pathway. LPS, lipopolysaccharide; TGF- $\beta$, transforming growth factor- $\beta$.

antibody (1:1,000 dilution; cat. no. sc-371; Santa Cruz Biotechnology, Inc.), anti-p-IкB $\alpha$ antibody (1:200 dilution; cat. no. sc-8404; Santa Cruz Biotechnology, Inc.) and anti-GAPDH (1:5,000 dilution; cat. no. ab9485; Abcam). After two washes with 1x TBS, the membranes were incubated with a horseradish peroxidase-conjugated secondary antibody (1:1,000, cat. no. 7074; Cell Signaling Technology, Inc.) for $2 \mathrm{~h}$ at room temperature, and the protein levels were detected using ECL western blotting reagents (cat. no. RPN2135, GE Healthcare) and Image lab v3.0 software (Bio-Rad Laboratories, Inc.).

Statistical analysis. Data are presented as the mean \pm the standard error of the mean. GraphPad Prism version 5 (GraphPad Software, Inc.) was used to perform the statistical analysis. Differences between two group were compared using a Student's t-test, and the differences among multiple groups were analyzed using a one-way ANOVA. All experiments were repeated three times. $\mathrm{P}<0.05$ was considered to indicate a statistically significant difference.

\section{Results}

LPS injection induces inflammation and cardiac damage. To understand the molecular mechanism of sepsis, LPS was injected into mice to generate a sepsis model. Furthermore, an RNA-Seq-based transcriptome study was performed to understand the molecular basis of this illness. Before performing RNA-Seq, the effects of LPS on heart tissue damage were examined in mice at different time points. After 1, 2, 3 and 4 days of LPS injection, the heart tissue was sampled, and the inflammatory response was examined by western blot analysis. The results showed that PAI-1 and TNF- $\alpha$ levels were induced by LPS injection in heart tissue, and the highest levels were detected in the 4-day sample; therefore, the subsequent experiments were performed using 4-day LPS-injected mouse hearts (Fig. 1A). After 4 days of LPS injection, the heart tissue began to show an abnormal structure with aberrant cardiac muscle arrangement. Histopathological analysis was performed using HE staining. The results showed major cardiac disorder in LSP-injected hearts, and the symp- 

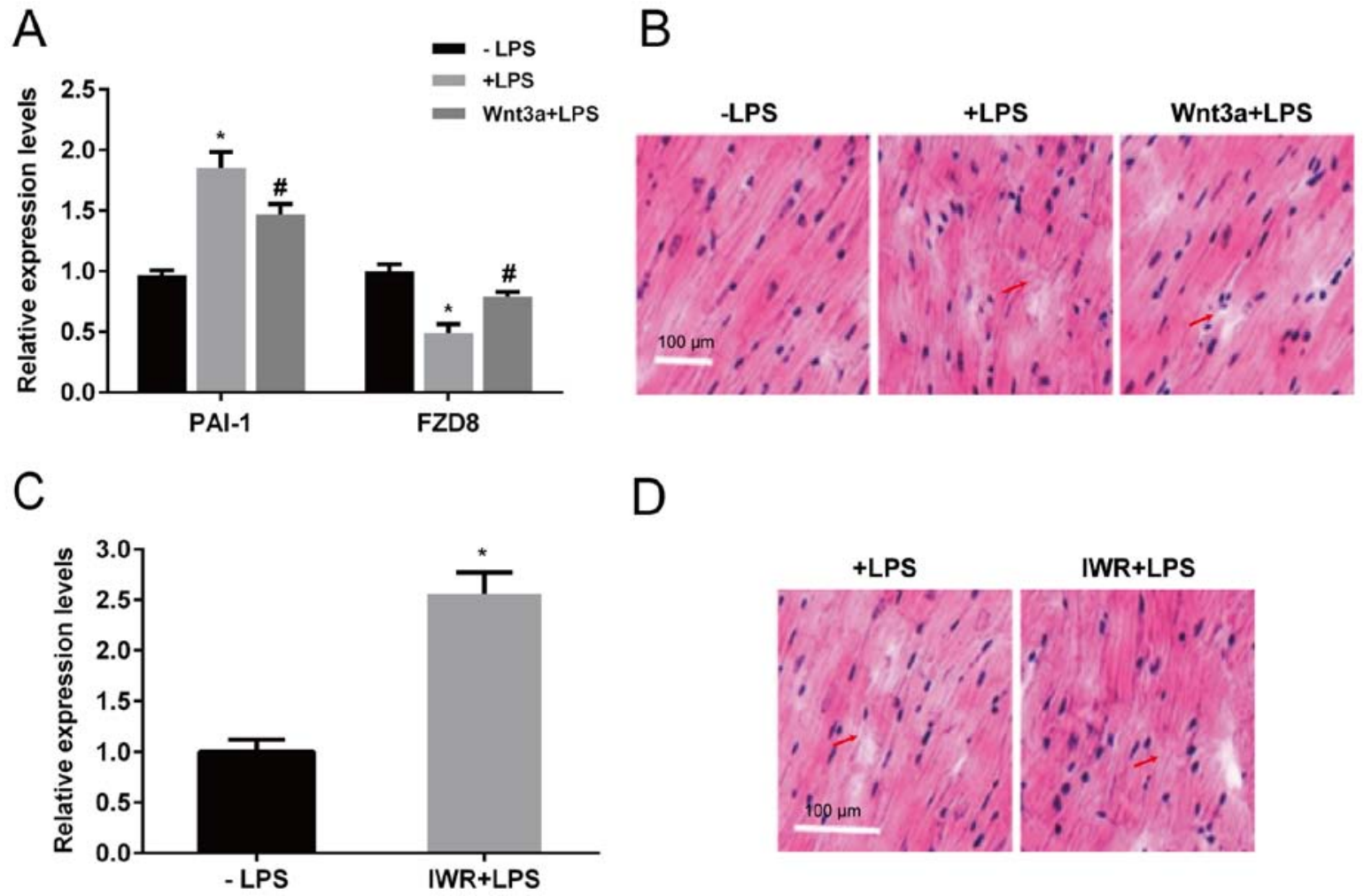

Figure 5. Effects of Wnt3a and IWR on expression of gene markers of heart tissue structure. LPS or Wnt3a together with LPS was injected, and the mouse hearts were extracted. (A) PAI-1 and FZD8 expression levels were determined by reverse transcription-quantitative PCR, and (B) heart tissue structure was analyzed by staining tissues with hematoxylin and eosin and observing under a light microscope. Scale bar, $100 \mu \mathrm{m}$. IWR, a Wnt signaling inhibitor, was injected, and (C) the PAI-1 expression level and (D) heart tissue structure were analyzed. Arrows indicated sites of myocardial fiber fracture. Scale bars, $100 \mu \mathrm{m}$. ${ }^{*} \mathrm{P}<0.05$ vs. the control group; ${ }^{*} \mathrm{P}<0.05$ vs. the LPS group. LPS, lipopolysaccharide; PAI-1, plasminogen activator inhibitor $1 ; F Z D 8$, frizzled class receptor 8 .

toms included increased disorganization and discontinuation of the myocardial structure (Fig. 1B). In addition, the levels of inflammatory responsive proteins were assessed using western blot analysis. The three inflammation-responsive markers tested, IL-6, IкB $\alpha$ and $\mathrm{p}-\mathrm{I} \kappa \mathrm{B}$, exhibited higher levels in LPS-injected mouse hearts compared with the control group (without injection) (Fig. 1C). These results suggest that LPS injection induces inflammatory responses and cardiac damage in mice.

LPS treatment alters the expression of several genes. To further investigate the molecular mechanism underlying sepsis illness, RNA-Seq experiments were carried out using mouse heart tissues with or without LPS injection for 4 days. The RNA-Seq results demonstrated that 5,094 genes were significantly altered by LPS ( $>$ two-fold changes; $\mathrm{P}<0.05$ ). Among them, 3,326 and 1,769 genes were upregulated and downregulated, respectively, by LPS injection in mouse hearts (Fig. 2A). The following genes were assessed by RT-qPCR to confirm the RNA-Seq data: Frizzled class receptor 8 (FZD8), $I L-8$, $P A I 1$ and fibroblast growth factor 21 (FGF21). The inflammatory response genes (IL-8 and PAI- 1 ) and FGF21 were upregulated, whereas a Wnt signaling gene (FZD8) decreased after LPS stimulation, and the RT-qPCR results were similar to the RNA-Seq data (Fig. 2B).

Differentially expressed genes are classified into diverse biological categories. In total, 5,094 differentially expressed genes regulated by LPS were further classified into GO and KEGG terms. GO analysis demonstrated that $41 \mathrm{GO}$ terms were highly enriched $(\mathrm{P}<0.01$; Fig. 3$)$. These genes are involved in multiple biological processes, including 'developmental process', 'cell part', 'binding' and 'cellular process' (Fig. 3). Several genes were enriched in the 'cell', 'cell part' and 'cellular process' biological categories. Subsequently, pathway analysis was performed using the 5,094 genes that were differentially expressed by LPS damage. The results showed that these genes were classified into 20 different pathways, such as the 'Wnt signaling pathway', 'VEGF signaling pathway', 'small cell lung cancer', and 'cardiac muscle contraction' pathways (Fig. 4).

Wnt signaling is involved in LPS-induced heart damage. As Wnt signaling genes were regulated by LPS injection in mice, the role of Wnt signaling in sepsis development was further investigated. Wnt signaling serves a key role in cell survival, proliferation and differentiation (25). To test the role of Wnt signaling in sepsis regulation, Wnt3a, an inducer of Wnt signaling, and IWR, an inhibitor of Wnt signaling were used. First, an inducer of Wnt signaling was injected into mice to determine the function of Wnt signaling in LPS-induced heart damage. After 4 days of injection, LPS induced PAI-1 expression, but Wnt3a treatment partially inhibited the LPS-mediated induction of PAI-1. In addition, LPS treatment suppressed $F Z D 8$ expression, whereas Wnt3a injection inhibited the LPS suppression of FZD8 (Fig. 5A). Heart tissues were 
evaluated further by HE staining. LPS injection damaged heart tissues by rearranging muscle, which exhibited deconstruction, whereas Wnt3a protected against LPS-induced cardiac damage (Fig. 5B). In contrast, IWR, a Wnt signaling inhibitor, was injected into the mice. After 4 days of injection, PAI-1 expression was significantly induced in the heart (Fig. 5C). Furthermore, IWR injection damaged heart tissues, similar to LPS injection, which exhibited aberrant cardiac muscle (Fig. 5D).

\section{Discussion}

Sepsis is caused by the imbalanced response of humans to pathogen infection, and this may result in severe damage to various organs, including the heart. A previous clinical study demonstrated that sepsis during the early stages is associated with a high incidence of organic damage to the myocardium, which causes hypotension, heart failure and arrhythmia (42). Currently, $40 \%$ of patients with sepsis suffer from myocardial injury, $\sim 60 \%$ of patients in intensive care units have clinical manifestations of myocardial injury, and the mortality of patients with sepsis can reach 70-90\% (43). However, no therapeutic strategies are available for patients with sepsis, and therefore, this issue has attracted attention worldwide. Exploring the molecular basis of sepsis will be useful for isolating target genes or proteins for therapy. However, studies regarding sepsis are still limited to date.

In the present study, bacterial endotoxin, including LPS, which is a potential inducer of inflammation, was injected into a mouse model of sepsis, and transcriptome analysis was performed to understand the molecular mechanism of sepsis in the mouse heart model. Initially, LPS was injected; which markedly induced an inflammatory response, based on evaluation of inflammatory response markers by western blot analysis. Additionally, histological analysis demonstrated that LPS injection severely damaged cardiac tissues by rearranging muscle order. This may have been caused by either the injection of low-concentration LPS or the LPS tolerance response (44); however, further analyses are required to verify this observation. The inflammatory response was also induced by the detection of IL-6, I $\mathrm{B} \alpha$, $\mathrm{p}-\mathrm{I} \kappa \mathrm{B} \alpha, \mathrm{PAI}-1$ and TNF- $\alpha$ levels using western blot analysis. LPS treatment significantly increased the expression levels of these protein, The critical step in NF-kB activation is the phosphorylation of $\operatorname{IkB} \alpha$ (45), suggesting that TNF- $\alpha$ and $\mathrm{NF}-\kappa \mathrm{B}$ signaling was activated in the mouse heart. In a subsequent RNA-seq-based transcriptome study, the expression levels of several genes were altered by injection of LPS in mouse hearts. Among these, 3,326 and 1,769 genes were upregulated and downregulated, respectively. These differentially expressed genes were classified into $41 \mathrm{GO}$ terms and 20 KEGG pathways. Among the 20 enriched pathways, 'Wnt signaling pathway', 'VEGF signaling pathway', 'TFG- $\beta$ signaling pathway' and 'cytokine-cytokine receptor interaction' pathways, and activity of certain metabolic pathways was altered. The western blot analysis suggested that TNF- $\alpha$ and $\mathrm{NF}-\kappa \mathrm{B}$ signaling was significantly increased by LPS injection. However, these signaling pathway genes were not enriched in the KEGG analysis. This difference in results may be due to the induction time point for transcripts or proteins, as 4 days is a relatively long period, and the occurrence of transcriptional changes is normally fast, or the increase in the number of these pathway genes may be lower. Nevertheless, $I L-8$ was detected and induced by LPS treatment. TNF- $\alpha$ and IL proteins are cytokines that serve important roles during the inflammatory response (46), and the KEGG analysis also showed that the cytokine-cytokine receptor interaction term was enriched. VEGF, TGF- $\beta$ and small lung cancer pathways are known to serve roles during cancer development $(47,48)$. Notably, another key signaling pathway in cancer biology, the Wnt signaling pathway, was enriched, and the Wnt genes were suppressed by LPS treatment, suggesting that these signaling pathways may be associated with inflammation and sepsis. Furthermore, the role of Wnt signaling was analyzed by activation or inhibition experiments in the present study. The function of Wnt signaling in sepsis has not been previously reported to the best of our knowledge. Furthermore, Wnt3a was added to induce Wnt signaling, to determine the role of Wnt signaling during sepsis. Wnt3a treatment inhibited LPS-mediated PAI- 1 and FZD 8 gene expression, and also prevented damage to cardiac tissue structure caused by LPS. Wnt3a treatment together with LPS reduced the LPS-induced damage to the myocardium. A Wnt signaling inhibitor, IWR, was subsequently injected; IWR injection increased the PAI-I expression levels and resulted in damage to the heart tissues, which exhibited abnormal cardiac organization. These data suggest that Wnt signaling protects against LPS-induced heart damage by reducing the inflammatory response. In addition, LPS treatment reduced expression of the Wnt signaling gene $F Z D 8$, suggesting that the LPS-mediated inflammatory response signaling may function upstream of Wnt signaling. Wnt3a and IWR treatment also inhibited and induced $P A I-1$ expression, respectively, suggesting that Wnt signaling functioned upstream of the inflammatory responsive gene $P A I-1$. These data suggest that Wnt and inflammatory response signals are associated with each other, and the crosstalk should be further analyzed in future studies. FGF21, a fibroblast growth factor, was also induced by LPS damage. FGF21 is an important hormone that protects organs from different damages $(49,50)$. Testing FGF21 function in further studies may be useful. However, the present study has its own limitations. Key regulators of Wnt signaling GSK-3 $\beta$ and $\beta$-catenin, were not measured following treatment with LPS, Wnt3a and IWR.

In conclusion, RNA-Seq analysis was performed to determine the molecular mechanism of a sepsis-damaged heart using a mouse model generated by LPS injection. The data suggested that sepsis damage in the heart may be caused by complicated processes, but LPS inhibited Wnt signaling to induce heart injury. This finding may be important for further exploration of sepsis-mediated damage to different tissues.

\section{Acknowledgements}

Not applicable.

\section{Funding}

No funding was received. 


\section{Availability of data and materials}

The datasets generated during the current study are not publicly available because the investigator team is still actively analyzing and publishing study results, but are available from the corresponding author on reasonable request.

\section{Authors' contributions}

$\mathrm{CC}, \mathrm{JW}$ and DF performed the experiments, wrote the manuscript, as well as the analysis and interpretation of data. JC collected and analyzed the data. MC designed the study. All authors read and approved the final manuscript.

\section{Ethics approval and consent to participate}

All animal studies were performed according to international ethical guidelines and the National Institutes of Health Guide Concerning the Care and Use of Laboratory Animals with The Approval of The Animal Experimentation Ethics Committee of The Affiliated Hospital of Putian University.

\section{Patient consent for participation}

Not applicable.

\section{Competing interests}

The authors declare that they have no competing interests.

\section{References}

1. Michalek SM, Moore RN, McGhee JR, Rosenstreich DL and Mergenhagen SE: The primary role of lymphoreticular cells in the mediation of host responses to bacterial endotoxim. J Infect Dis 141: 55-63, 1980.

2. Beeson PB and Technical Assistance of Elizabeth Roberts: Tolerance to bacterial pyrogens: I. Factors influencing its development. J Exp Med 86: 29-38, 1947.

3. Singer M, Deutschman CS, Seymour CW, Shankar-Hari, M, Annane D, Bauer, M, Bellomo, R, Bernard GR, Chiche JD, Coopersmith CM, et al: The third international consensus definitions for sepsis and septic shock (sepsis-3). JAMA 315: 801-810, 2016.

4. Seymour CW, Liu VX, Iwashyna TJ, Brunkhorst FM, Rea TD, Scherag A, Rubenfeld G, Kahn JM, Shankar-Hari M, Singer M, et al: Assessment of clinical criteria for sepsis: For the third international consensus definitions for sepsis and septic shock (sepsis-3). JAMA 315: 762-774, 2016.

5. Engel C, Brunkhorst FM, Bone HG, Brunkhorst R, Gerlach $\mathrm{H}$, Grond S, Gruendling M, Huhle G, Jaschinski U, John S, et al Epidemiology of sepsis in Germany: Results from a national prospective multicenter study. Intensive Care Med 33: 606-618, 2007.

6. Angus DC and Wax RS: Epidemiology of sepsis: An update. Crit Care Med 29 (7 Suppl): S109-S116, 2001.

7. Cohen J: The immunopathogenesis of sepsis. Nature 420: 885-891, 2002.

8. Drosatos K, Lymperopoulos A, Kennel PJ, Pollak N, Schulze PC and Goldberg IJ: Pathophysiology of sepsis-related cardiac dysfunction: Driven by inflammation, energy mismanagement, or both? Curr Heart Fail Rep 12: 130-140, 2015.

9. Harbarth S, Holeckova K, Froidevaux C, Pittet D, Ricou B, Grau GE, Vadas L and Pugin J; Geneva Sepsis Network: Diagnostic value of procalcitonin, interleukin-6, and interleukin-8 in critically ill patients admitted with suspected sepsis. Am J Respir Crit Care Med 164: 396-402, 2001.

10. Simon L, Gauvin F, Amre DK, Saint-Louis P and Lacroix J: Serum procalcitonin and C-reactive protein levels as markers of bacterial infection: A systematic review and meta-analysis. Clin Infect Dis 39: 206-217, 2004.
11. Bozza FA, Salluh JI, Japiassu AM, Soares M, Assis EF, Gomes RN, Bozza MT, Castro-Faria-Neto HC and Bozza PT: Cytokine profiles as markers of disease severity in sepsis: A multiplex analysis. Crit Care 11: R49, 2007.

12. Vaschetto R, Nicola S, Olivieri C, Boggio E, Piccolella F, Mesturini R, Damnotti F, Colombo D, Navalesi P, Della Corte F, et al: Serum levels of osteopontin are increased in SIRS and sepsis. Intensive Care Med 34: 2176-2184, 2008.

13. Brenner T, Rosenhagen C, Steppan J, Lichtenstern C, Weitz J, Bruckner T, Martin EO, Hoffmann U, Weigand MA and Hofer S: Redox responses in patients with sepsis: High correlation of thioredoxin- 1 and macrophage migration inhibitory factor plasma levels. Mediators Inflamm 2010: 985614, 2010.

14. Bae JS: Role of high mobility group box 1 in inflammatory disease: Focus on sepsis. Arch Pharm Res 35: 1511-1523, 2012.

15. Wu Y, Wang F, Fan X, Bao R, Bo L, Li J and Deng X: Accuracy of plasma sTREM-1 for sepsis diagnosis in systemic inflammatory patients: A systematic review and meta-analysis. Crit Care 16: R229, 2012.

16. Backes Y, van der Sluijs KF, Mackie DP, Tacke F, Koch A, Tenhunen JJ and Schultz MJ: Usefulness of suPAR as a biological marker in patients with systemic inflammation or infection: A systematic review. Intensive Care Med 38: 1418-1428, 2012.

17. Rautanen A, Mills TC, Gordon AC, Hutton P, Steffens M, Nuamah R, Chiche JD, Parks T, Chapman SJ, Davenport EE, et al: Genome-wide association study of survival from sepsis due to pneumonia: An observational cohort study. Lancet Respir Med 3: 53-60, 2015.

18. Scherag A, Schöneweck F, Kesselmeier M, Taudien S, Platzer M, Felder M, Sponholz C, Rautanen A, Hill AVS, Hinds CJ, et al: Genetic factors of the disease course after sepsis: A genome-wide study for 28 day mortality. EbioMedicine 12: 239-246, 2016.

19. Wang L, Ko ER2, Gilchrist JJ, Pittman KJ, Rautanen A, Pirinen M, Thompson JW, Dubois LG, Langley RJ, Jaslow SL, et al: Human genetic and metabolite variation reveals that methylthioadenosine is a prognostic biomarker and an inflammatory regulator in sepsis. Sci Adv 3: e1602096, 2017.

20. Brown MA and Jones WK: NF-kappaB action in sepsis: The innate immune system and the heart. Front Biosci 9: 1201-1217, 2004.

21. Li Y, Ge S, Peng Y and Chen X: Inflammation and cardiac dysfunction during sepsis, muscular dystrophy, and myocarditis. Burns Trauma 1: 109-121, 2013.

22. MacDonald BT, Tamai K and He X: Wnt/beta-catenin signaling: Components, mechanisms, and diseases. Dev Cell 17: 9-26, 2009.

23. Gordon MD and Nusse R: Wnt signaling: Multiple pathways, multiple receptors, and multiple transcription factors. J Biol Chem 281: 22429-22433, 2006.

24. Shtutman M, Zhurinsky J, Simcha I, Albanese C, D'Amico M, Pestell $\mathrm{R}$ and Ben-Ze'ev A: The cyclin D1 gene is a target of the beta-catenin/LEF-1 pathway. Proc Natl Acad Sci USA 96: 5522-5527, 1999.

25. Moon RT, Kohn AD, De Ferrari GV and Kaykas A: WNT and $\beta$-catenin signalling: Diseases and therapies. Nat Rev Genet 5: 691-701, 2004.

26. Clevers $\mathrm{H}$ : Wnt/ $\beta$-catenin signaling in development and disease. Cell 127: 469-480, 2006.

27. Brack AS, Conboy MJ, Roy S, Lee M, Kuo CJ, Keller C and Rando TA: Increased Wnt signaling during aging alters muscle stem cell fate and increases fibrosis. Science 317: 807-810, 2007.

28. Aberle H, Bauer A, Stappert J, Kispert A and Kemler R: Beta-catenin is a target for the ubiquitin-proteasome pathway. EMBO J 16: 3797-3804, 1997.

29. National Research Council: Guide for the care and use of laboratory animals. National Academies Press, Washington, DC, 1996.

30. Leary S: AVMA Guidelines for the Euthanasia of Animals: 2013 Edition. American Veterinary Medical Association. J Am Veterinary Med Association, 2013.

31. Livak KJ and Schmittgen TD: Analysis of relative gene expression data using real-time quantitative PCR and the 2(-Delta Delta C(T)) method. Methods 25: 402-408, 2001.

32. Rani B: RNA Sequencing (RNA-Seq): Method and Applications. Int J Pure App Biosci 6: 167-173, 2018.

33. Kim D, Pertea G, Trapnell C, Pimentel H, Kelley R and Salzberg SL: TopHat2: Accurate alignment of transcriptomes in the presence of insertions, deletions and gene fusions. Genome Biol 14: R36, 2013.

34. Langmead B and Salzberg SL: Fast gapped-read alignment with Bowtie 2. Nat Methods 9: 357-359, 2012. 
35. Gene Ontology Consortium. The gene ontology (GO) project in 2006. Nucleic Acids Res 34: D322-D326, 2006.

36. Dupuy D, Bertin N, Hidalgo CA, Venkatesan K, Tu D, Lee D, Rosenberg J, Svrzikapa N, Blanc A, Carnec A, et al: Genome-scale analysis of in vivo spatiotemporal promoter activity in Caenorhabditis elegans. Nat Biotechnol 25: 663-668, 2007.

37. Dunnick JK, Brix A, Cunny H, Vallant M and Shockley KR: Characterization of polybrominated diphenyl ether toxicity in Wistar Han rats and use of liver microarray data for predicting disease susceptibilities. Toxicol Pathol 40: 93-106, 2012.

38. Kanehisa M, Goto S, Kawashima S and Nakaya A: The KEGG databases at Genome Net Nucleic Acids Res 30: 42-46, 2002.

39. Nishimura D: BioCarta. Biotech Software Internet Rep 2: $117-120,200$

40. Matthews L, Gopinath G, Gillespie M, Caudy M, Croft D, de Bono B, Garapati P, Hemish J, Hermjakob H, Jassal B, et al: Reactome knowledgebase of human biological pathways and processes. Nucleic Acids Res 37 (Database Issue): D619-D622, 2009.

41. Draghici S, Khatri P, Tarca AL, Amin K, Done A, Voichita C, Georgescu C and Romero R: A systems biology approach for pathway level analysis. Genome Res 17: 1537-1545, 2007.

42. Turner A, Tsamitros M and Bellomo R: Myocardial cell injury in septic shock. Crit Care Med 27: 2035-2036, 1999.

43. Hochstadt A, Meroz Y and Landesberg G: Myocardial dysfunction in severe sepsis and septic shock: More questions than answers? J Cardiothorac Vasc Anesth 25: 526-535, 2011.

44. Dios S, Balseiro P, Costa MM, Romero A, Boltaña S, Roher N, Mackenzie S, Figueras A and Novoa B: The involvement of cholesterol in sepsis and tolerance to lipopolysaccharide highlighted by the transcriptome analysis of zebrafish (Danio rerio). Zebrafis 11: 421-433, 2014.
45. Traenckner EB, Pahl HL, Henkel T, Schmidt KN, Wilk S and Baeuerle PA: Phosphorylation of human I kappa B-alpha on serines 32 and 36 controls I kappa B-alpha proteolysis and NF-kappa B activation in response to diverse stimuli. EMBO J 14: 2876-2883, 1995

46. Jaffer U, Wade RG and Gourlay T: Cytokines in the systemic inflammatory response syndrome: A review. HSR Proc Intensive Care Cardiovasc Anesth 2: 161-175, 2010.

47. Carmeliet P: VEGF as a key mediator of angiogenesis in cancer. Oncology 69: 4-10, 2005.0

48. Derynck R, Akhurst RJ and Balmain A: TGF- $\beta$ signaling in tumor suppression and cancer progression. Nature Genet 29: 117-129, 2001.

49. Planavila A, Redondo-Angulo I, Ribas F, Garrabou G, Casademont J, Giralt $\mathrm{M}$ and Villarroya F: Fibroblast growth factor 21 protects the heart from oxidative stress. Cardiovasc Res 106: 19-31, 2015.

50. Singhal G, Fisher FM, Chee MJ, Tan TG, El Ouaamari A, Adams AC, Najarian R, Kulkarni RN, Benoist C, Flier JS and Maratos-Flier E: Fibroblast Growth Factor 21 (FGF21) protects against high fat diet induced inflammation and islet hyperplasia in pancreas. PLoS One 11: e0148252, 2016.

This work is licensed under a Creative Commons Attribution-NonCommercial-NoDerivatives 4.0 International (CC BY-NC-ND 4.0) License. 